\title{
El derecho a la salud es un derecho humano
}

The right to health is a human right

Esther Illán Marcos, ${ }^{*}$ César Hernández Mier ${ }^{\ddagger}$

\section{RESUMEN}

En este artículo se formula una presentación del derecho a la salud con perspectiva de derechos humanos. Se considera importante que se realicen modificaciones en el Sistema Nacional de Salud mexicano a fin de ampliar el acceso a los servicios sanitarios. El Instituto de Salud para el Bienestar (INSABI) se ostenta como un avance en protección social pero no mejora la condición de sanidad de la población, debido a que el Sistema Nacional de Salud se encuentra fragmentado y debería modificarse la estructura. También es fundamental que se atienda a factores socioeconómicos y otras aristas que presentan vinculación directa con la sanidad, debido a que los derechos de la humanidad son una unidad y si se atienden individualmente se produce la deficiencia de todos, tanto en su protección como en su desarrollo efectivo.

Palabras clave: Derecho a la salud, derecho a la protección de la salud, derechos humanos.

\section{ABSTRACT}

This article formulates the right to health from a human rights perspective. It is considered important that modifications be made to the Mexican National Health System to expand access to health services. INSABI is boasted as an advance in social protection but it does not improve the health condition of the people, because the National Health System is fragmented and the structure must be modified. It is also essential that socio-economic factors and other rights that are directly linked to health be addressed, since human rights are a unit.

Keywords: Right to health, right to health protection, human rights.

* Maestra en Derechos
Humanos.
₹ Profesor Investigador.

Facultad de Derecho «Abogado Ponciano Arriaga Leija» de la Universidad Autónoma de San Luis Potosí, México.

Correspondencia: EIM, esther.yllan.marcos@ gmail.com

Conflicto de intereses: No existe conflicto de intereses. (No pertenezco a ningún partido político o sindicato).

Citar como: Illán ME, Hernández MC. El derecho a la salud es un derecho humano. Rev CONAMED. 2021; 26(2): 89-94. https:// dx.doi.org/10.35366/100351 Financiamiento: No tuvo financiación.

Recibido: 09/10/2020. Aceptado: 03/06/2021. 


\section{INTRODUCCIÓN}

El cumplimiento del derecho a la salud es un elemento fundamental que persigue el bienestar de las personas. La desatención de esta facultad tiene una repercusión negativa en otros derechos humanos debido a los principios de indivisibilidad e interdependencia. Con la meta de comprender mejor las vulneraciones que suceden en el derecho a la sanidad, se ha considerado relevante escribir este trabajo, de manera sucinta, en él se pretende exponer esta facultad como un derecho humano y señalar la trascendencia que tiene su correcta comprensión para mejorar las carencias que han quedado demostradas en el Sistema Nacional de Salud en México. Se reivindica que es de gran relevancia que se formulen nuevas reformas en el Sistema Nacional de Salud y así poder ampliar el acceso a los servicios de salud. También se mencionan de manera concisa las transgresiones a los derechos humanos durante la gestión de la pandemia en Latinoamérica. El desarrollo de la presente investigación se realizó a través de un diseño de tipo exploratorio, mediante la hermenéutica jurídica.

\section{EL DERECHO A LA SALUD Y SUS ELEMENTOS}

La constitución de la Organización Mundial de la Salud (OMS) entró en vigor el 7 de abril de 1948. En su preámbulo, se puede encontrar que la salud es «un estado de completo bienestar físico, mental y social, y no solamente la ausencia de afecciones o enfermedades». Al tomar como base esta definición, la OMS ha considerado dicha normativa como «el goce del grado máximo de salud que se puede lograr» y debe ser entendido como «uno de los derechos fundamentales de todo ser humano sin distinción de raza, religión, ideología política, condición económica o social». Tal facultad no debería ser interpretada como la exigencia del pueblo hacia el gobierno de estar siempre «sano», lo cual sería inalcanzable, nunca habría de olvidarse que el ser humano es perecedero. Por tanto, en la situación actual de pandemia, no se podría exigir que tras la enfermedad COVID-19 los individuos no tengan secuelas. Lo que sí se puede reivindicar es la garantía de recibir una vacuna y a ser atendido respetando la dignidad y sin discriminación alguna.
Es importante conocer que la mencionada potestad abarca cuatro piezas, que son: disponibilidad, accesibilidad, aceptabilidad y calidad. Cada una de ellas definida por la Comisión Nacional de Derechos Humanos del siguiente modo:

1. La disponibilidad contempla el contar con el número suficiente de establecimientos, bienes, insumos y servicios.

2. La accesibilidad es definida como la obligación de que los establecimientos de atención médica estén al alcance de todos y la no discriminación.

3. La aceptabilidad significa que los servicios deben proporcionar una atención con enfoque diferencial y especializado, respetando los aspectos culturales y sensibles de los requisitos de cada grupo y cada paciente.

4. La calidad ha de encontrarse tanto en la educación y formación de profesionales de salud al igual que en las instalaciones y materiales empleados.

Por lo anterior, cada gobierno debe establecer acciones encaminadas a permitir que sus ciudadanos cuenten con acceso al sistema de salud con todos sus elementos mencionados. Lo hacen a través de la positivización de esta garantía. El derecho humano a la salud es internacional y universal; cuando cada país lo incluye en su propio ordenamiento jurídico lo nacionaliza y lo convierte en derecho positivo de su estado. A nivel internacional, esa facultad se encuentra protegida por diversos instrumentos, por ejemplo la Declaración Universal de los Derechos Humanos (DUDH) en su artículo 25. Mientras que en América Latina lo está por la Convención Americana de Derechos Humanos, entre otros textos. En particular, en México se encuentra resguardado por el artículo $4^{\circ}$ de la Constitución Política de los Estados Unidos Mexicanos, ${ }^{2}$ estableciendo que «Toda persona tiene derecho a la protección de la salud», sin embargo, es la Ley General de Salud la que establece las bases y modalidades de este acercamiento.

Lo anterior muestra que, en materia del derecho humano a la salud, existe una fortaleza legislativa que positiviza la prerrogativa y la protege, aunque en la práctica se identifiquen deficiencias en el cumplimiento. Tras percatarse de esta situación, el Comité de Derechos Económicos, 
Sociales y Culturales decidió emitir su Observación $N^{\circ} 14^{3}$ donde señala que la mencionada garantía incluye: Ios derechos a un sistema de protección de la salud, a la prevención, a recibir diagnóstico y tratamiento, el ingreso a la educación que permita formar a los profesionales y también poder educar a la población a modo de que disponga de conocimientos básicos para saber los derechos que les asisten.

\section{EL DERECHO A LA SALUD Y LOS PRINCIPIOS GENERALES DE LOS DERECHOS HUMANOS}

La Corte Interamericana, en su Cuaderno № 28 de Jurisprudencia, ha querido reseñar algunos pronunciamientos en relación con los principios de derechos humanos que están vinculados con el derecho a la salud y ha determinado que éstos son tres: de progresividad, de indivisibilidad y de interdependencia.

\section{El principio de progresividad}

El derecho humano a la salud es considerado de la segunda generación, es decir, forma parte de la categoría de los conocidos como Derechos Económicos, Sociales, Culturales y Ambientales (DESCA). Pertenecer a esta categoría tiene el inconveniente de estar condicionado a las posibilidades económicas de cada nación para poder proporcionar su efectivo cumplimiento. Desafortunadamente ese derecho a la salud, y en consecuencia a la vida, resulta condicionado a la economía del paciente y del país donde resida. Esta afirmación ha quedado reflejada en el creciente turismo sanitario, que consiste en incluir la posibilidad de vacunarse contra la COVID-19 al viajar a otro territorio. Esta práctica se está llevando a cabo por ejemplo en Maldivas, Alaska, Rusia y Serbia, donde of recen a los turistas la posibilidad de vacunarse, todo organizado por las agencias de viajes. Cada vez se suman más naciones a esta nueva iniciativa y la salud se está mercantilizando a nivel global de un modo que nunca antes lo había hecho.

El principio de progresividad reconoce una cierta gradualidad hacia su realización, pero nunca se le debería utilizar como argumento de retrocesos en el desarrollo y protección de derechos humanos. ${ }^{4}$ Siempre se habría de tender a ir hacia el progreso, dentro de las posibilidades económicas. Se requiere la mejora efectiva de las condiciones hacia el goce y el ejercicio del derecho a la salud. Conviene poner especial atención a corregir las desigualdades sociales y a que se facilite la inclusión de los grupos vulnerados en los sistemas nacionales en esa materia; de manera que queda totalmente prohibida la inactividad de los estados ante su tarea de ir implementando acciones que protejan esta normatividad.

El precepto expuesto se podría apreciar, por ejemplo, cuando en la compra de material hospitalario durante la pandemia; las cifras varían mucho de un país a otro y eso se debe a la economía de que dispone cada región. De tal manera, al gobierno mexicano no se le puede recriminar que no haya invertido o comprado las mismas cifras que, por ejemplo, Francia. Siempre y cuando el gobierno mexicano pueda demostrar que ha actuado acorde a su posibilidad económica, no se considerará que ha transgredido este precepto.

\section{Los principios de indivisibilidad e interdependencia}

Los derechos humanos son un todo, una unidad que se encuentra formada por diversas normas. Tales normas se dividen en generaciones para facilitar su estudio y enseñanza, pero en realidad no existe jerarquía entre ellos, todos tendrían que ser respetados y atendidos por igual. Ahí radicaría el problema de los gobiernos que normalmente, cuando realizan una distribución presupuestaria, dan preferencia a unos derechos frente a otros. De esta forma, en la práctica ningún derecho de los individuos es atendido de manera eficiente, porque al ser desatendidos unos, se causa deficiencias en los demás.

\section{RELACIÓN ENTRE EL DERECHO A LA SALUD Y OTROS DERECHOS HUMANOS DURANTE LA PANDEMIA}

Al tener en cuenta los principios de indivisibilidad e interdependencia se puede comprender mejor la situación actual que se está viviendo a nivel mundial. Se han visto afectados múltiples derechos y no únicamente el de la salud. De manera breve, a continuación se procede a exponer algunas 
vulneraciones a los derechos humanos durante la gestión de la pandemia en Latinoamérica.

En 2021 los países empezaron a analizar con detalle y a enfrentar los daños económicos causados por la pandemia y se han creado tensiones sociales cada vez mayores tanto a nivel nacional como internacional.

En abril de 2021, en la Cumbre Iberoamericana de Jefes de Estado, celebrada en Andorra, estos líderes partieron de la preocupación de la caída del producto interno bruto (PIB) superior al 7\% en Latinoamérica. Una de las consecuencias de la pandemia está siendo la pobreza económica y el desempleo. En definitiva, se está quebrantando el derecho a un trabajo digno. Con el aumento del desempleo y debido a las medidas de distanciamiento social, los primeros afectados fueron los miembros de las poblaciones más inermes. La pandemia sigue agravando la inopia de muchas familiasy, de manera recíproca, la miseria repercute en el aumento de los casos de contagio y fallecimientos. Esto es así debido a que la pobreza es un factor que transgrede la posibilidad del acceso a la salud. Ya que, si bien existe una cobertura garantizada y respaldada en las legislaciones locales y tratados internacionales, se continúa identificando cómo los ciudadanos con menos recursos económicos no acceden a una educación para la sanidad, presentan limitaciones al realizar traslados a los establecimientos de atención hospitalaria, y no cuentan con los recursos necesarios a fin de acceder al tratamiento y medicamentos no cubiertos. Es por ello que la Organización Mundial de la Salud ha formulado estudios importantes que relacionan la pobreza con la salud determinando que «la pobreza ha sido y seguirá siendo el mayor obstáculo al desarrollo de la salud».

Tras partir de esta afirmación, preocupa la situación postpandémica en Latinoamérica. La Comisión Económica para la América Latina y el Caribe ha señalado que la cantidad de gente que vive en situación de pobreza extrema ha aumentado y está alcanzando un nivel que no se veía desde hacía aproximadamente 20 años. Por lo tanto, en América Latina se ha retrocedido en bienestar y calidad de vida. Se contempla que disminuir la pobreza va a ser difícil en los próximos años, debido a que los países más ricos se muestran velando por sus intereses buscando recuperarse de las pérdidas económicas y de personas. En estos momentos, la solidaridad internacional está quedando rezagada. Un ejemplo es el caso de España, que se ha comprometido a enviar siete millones de vacunas a América Latina a través del mecanismo multilateral Covax y en colaboración con la Organización Panamericana para la Salud. Pero esto sucederá cuando alcance el $50 \%$ de su población vacunada, y en España, en la actualidad, se tiene alrededor de 38\%. Todavía habrán de pasar meses hasta que envíen las vacunas, y mientras, los niveles de fallecidos continúan subiendo.

Además de las infracciones al derecho a la salud, al trabajo y a la vida, se ha detectado un incremento de la violencia. El Programa de las Naciones Unidas para el Desarrollo (PNUD) ha publicado un informe que indica que la violencia de género y doméstica ha aumentado en América Latina y el Caribe durante la pandemia. Las estrictas medidas de permanecer en el hogar, la falta de empleo y el no poder asistir a los centros educativos, han generado dificultades en la convivencia de los hogares, obligando a las víctimas a vivir con sus agresores.

En la conclusión de este apartado se desea reflexionar sobre el derecho a la información, que también se está viendo afectado durante la pandemia. Es primordial informar a la ciudadanía acerca de los riesgos para la salud que representa la COVID-19, al igual que las medidas preventivas. El respeto a la libertad de expresión implica el derecho a dar a conocer opiniones e ideas, y deliberar abiertamente permite el acceso a la información. La cuestión es que una gran cantidad de personas acepta como veraz todo lo que circula en redes sociales e internet, y esto fomenta que muchas de ellas, por ejemplo, no quieran vacunarse. Los medios de comunicación tienen un papel fundamental en la prevención del pánico en la ciudadanía y ayudar a concientizar y generar mayor responsabilidad por parte de los ciudadanos en cumplir con las medidas preventivas.

En este texto se quiere pedir prudencia ante los datos a los que se podría llegar a tener acceso, con relación al número de contagios y fallecimientos de cada país, porque se ha detectado que los países que muestran las cifras más altas en Latinoamérica, son aquellos con mayor nivel de capacidad estadística, es decir, con más avance tecnológico y experiencia en el estudio de los datos; tienen 
mejores mecanismos que logran procesar y almacenar información sobre la población. Los estados más pobres en Latinoamérica, por ejemplo Guatemala, Bolivia y Honduras, en la clasificación de afectados se encuentran por debajo de Brasil, México y Chile. ¿Cómo puede ser posible que un factor como la pobreza no se haya visto reflejado en el número de afectados de los países más pobres de Latinoamérica? La conjetura propuesta es que no se sabe la realidad de los países más pobres porque no tienen los medios económicos ni tecnológicos a fin de reportar a nivel global sus datos en relación con la pandemia. No se sabe con exactitud cuántas personas han podido contagiarse y fallecer, de manera que el derecho a la información está siendo vulnerado por los diversos aspectos ya mencionados.

Todos las facultades listadas son ejemplos de cómo, al verse afectado un derecho humano, el derecho a la salud, han comenzado a resultar afectados otros. Existen muchos otros derechos que, aunque no han sido mencionados, no significa que no se estén viendo afectados también.

\section{EL DERECHO A LA SALUD EN MÉXICO}

Actualmente, en México se reconoce la salud como un derecho humano, fundamental y social. El artículo $4^{\circ}$ de la Constitución Política de los Estados Unidos Mexicanos dice así «Toda persona tiene derecho a la protección de la salud». ${ }^{5}$ El artículo fue creado de acuerdo con lo establecido en el artículo 25 de la DUDH. Es importante subrayar que dicha declaración no es vinculante para los estados, porque es una resolución de la Asamblea General de Naciones Unidas y los derechos humanos se encuentran limitados a que los países los incluyan en sus derechos vigentes.

En México, para hacer efectivo el artículo $4^{\circ}$ de la Constitución fue necesario desarrollarlo a través de la Ley General de Salud, la cual creó el Sistema Nacional de Salud, que se encuentra fragmentado atendiendo a la condición laboral y así atribuir unas prestaciones u otras. En 2019 se presentó una reforma al contenido de la Ley General de Salud, ${ }^{6}$ la cual consistía en proponer la disponibilidad universal en el sistema sanitario público mexicano, así se incluiría a todos aquellos que no estuvieran inscritos en la seguridad social y se crearía el Instituto de Salud para el Bienestar (INSABI); así, fue a inicios de 2020 cuando se produjeron los cambios en el Sistema Nacional de Salud. El INSABI es descrito como una institución que brindará atención médica «sin restricciones» al contemplar el acceso universal, mas no es lo mismo acceso que cobertura.

No se puede hablar de cobertura universal con relación al INSABI, porque está destinado a ofrecer solamente tratamientos de primer y segundo nivel, dejando el tercer nivel descubierto y correspondiendo al gasto personal y familiar. Respecto a la accesibilidad, tampoco se podría afirmar que el INSABI haya supuesto un cambio significativo, porque la accesibilidad implica igualmente vencer las dificultades geográficas que la población enfrenta intentando desplazarse desde su hogar hasta los hospitales y continúa existiendo escasez de ambulancias. De igual modo, las barreras para disponer de información son un impedimento a la accesibilidad, aún se necesita trabajar en crear folletos y documentación, por ejemplo, en Braille o en lenguas indígenas. Todo lo mencionado son ejemplos de motivos por los cuales debe considerarse que no es cierto que el INSABI cumpla con ser un mecanismo que brinda la universalidad en la disponibilidad a los servicios de sanidad, aunque se ostente como tal, si bien sí ha supuesto una ampliación de la población que logra tenerlo.

\section{CONCLUSIONES}

En opinión de los autores, la Agenda 2030 y otros pactos internacionales no verán cumplidos sus metas debido a que no se pudo prever la pandemia de COVID-19. La propagación del virus ha afectado la esfera de los derechos humanos a nivel planetario, dificultando la solidaridad internacional y los compromisos acordados.

A nivel nacional, los autores consideran que es recomendable un sistema nacional de salud unificado que permita optimizar los recursos existentes, y que del mismo modo permita a la ciudadanía poder acudir a cualquier hospital de la red de instituciones gubernamentales, es decir, que no exista distinción entre personas con seguridad social y aquellas sin ella, y puedan acceder en igualdad de condiciones a los mismos tratamientos y servicios. 
La organización actual genera la inexistencia de igualdad en el acceso y cobertura y la imposibilidad de lograr una disponibilidad universal.

A pesar de existir una normatividad robusta, siguen identificándose deficiencias en la atención médica y en el cumplimiento de esta atribución, siendo la pobreza uno de los mayores obstáculos hacia el desarrollo de la salud, y poniendo en peligro el bienestar, la vida de las personas, e incluso el crecimiento económico de una nación al debilitar su productividad e impactando en la pobreza, resultando esta última un factor que a su vez también impacta en la sanidad.

El citado derecho contempla elementos como la disponibilidad, accesibilidad, aceptabilidad y calidad, por lo que cada gobierno se encuentra obligado a establecer acciones que permitan dar cumplimiento a éstos.

De la misma manera, se identifica claramente que los ciudadanos con menos recursos económicos enfrentan limitaciones para acceder a acciones encaminadas a la educación y promoción de la salud, buscando acudir a los establecimientos de atención hospitalaria, y en su intento de acceder a tratamientos y medicamentos no cubiertos, por lo que la pobreza es el mayor obstáculo frente al desarrollo de la salud, con tal antecedente, se debe tener en cuenta el principio de progresividad en salud.

Se requiere reforzar los sistemas nacionales de salud mexicanos respetando el precepto de progresividad, y procurando asegurar el acceso de todas las personas a los servicios de salud, se recomienda fomentar la perspectiva de derechos humanos durante la formación de los profesionales del sector y realizar capacitaciones persiguiendo mejorar la relación médico-paciente. Es imperativo evaluar y reformar continuamente las estrategiasy planes de política pública de sanidad a fin de dar respuestas a las situaciones sanitarias del presente y futuro cercano.

\section{AGRADECIMIENTOS}

A todas las personas e instituciones que hayan contribuido en la realización de este artículo.

\section{REFERENCIAS}

1. Organización Mundial de la Salud. Constitución de la Organización Mundial de la Salud [Internet]. Suplemento de la 45 edición. 2006. Disponible en: https://www.who. int/governance/eb/who_constitution_sp.pdf

2. Cámara de Diputados. Constitución Política de los Estados Unidos Mexicanos [Internet]. 2020. Disponible en: http://www.diputados.gob.mx/LeyesBiblio/pdf_mov/ Constitucion_Politica.pdf

3. Naciones Unidas, Consejo Económico y Social, Comité de Derechos Económicos, Sociales y Culturales. Observación General Número 14, E/C. 12/2000/4.

4. De Lora P, Zúñiga F. El derecho a la asistencia sanitaria. Un análisis desde las teorías de la justicia distributiva. Madrid: Iustel; 2009. p. 19.

5. Cámara de Diputados del H. Congreso de la Unión. Constitución Política de los Estados Unidos Mexicanos; DOF 08-05-2020. Texto vigente; [Acceso Agosto de 2020]. Disponible en: www.diputados.gob.mx

6. Cámara de Diputados del H. Congreso de la Unión. Ley general de salud; DOF 24-01-2020. Texto vigente; [Acceso Agosto de 2020]. Disponible en: http://www.diputados.gob. $\mathrm{mx/LeyesBiblio/ref/lgs.htm}$

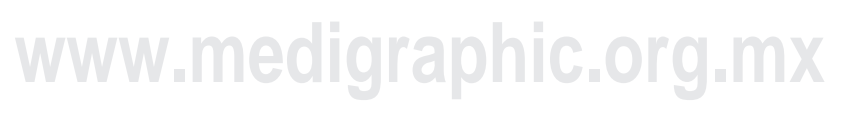

